\title{
Enantioseparation in CE using macrocyclic antibiotics as chiral selectors
}

\author{
F. Hui and M. Caude
}

Laboratoire Environnement et Chimie Analytique, associé au CNRS, ESPCI, 10 rue Vauquelin, 75231 Paris Cedex 05, France

\begin{abstract}
A great number of chiral selectors have been introduced for the CE enantioseparation and among them macrocyclic antibiotics constitute the newest class. To date, two types of macrocyclics show excellent enantioselectivity towards numerous racemates: the ansamycins and the glycopeptides. The former ones are particularly adept at resolving cationic compounds, while the later ones are high enantioselective for anionic compounds. In this paper, the structure and some physico-chemical properties of these macrocyclic antibiotics are presented. The effects of different operating parameters such as $\mathrm{pH}$, antibiotic type and concentration, chemical nature and concentration of the background electrolyte, organic modifier and micellar phase are discussed. Recent developments of the antibiotics-based enantioseparations are given as well.
\end{abstract}

\section{Introduction}

Enantiomer separation represents an important topic especially in pharmaceutical and environmental fields where many drugs and pesticides are racemic compounds. In some cases, only one of the enantiomers has pharmacological or pesticidal activity while the other may exhibit antagonistic activities, unwanted side effects or even toxic effects. Therefore, the development of new enantioseparation methods with high efficiency and sensitivity is of great interest for monitoring the stereoselective synthesis of enantiomers, pharmacological studies, enantiomeric purity control, environmental investigations, etc.
Capillary electrophoresis (CE) has proven to be an effective technique for chiral separation. As a complementary method to chromatographic techniques such as gas chromatography, liquid chromatography and more recently supercritical fluid chromatography. CE has some general merits including impressive peak efficiency, flexibility regarding choice of the chiral selectors and operating conditions, environmental friendliness, minimum consumption of reagents and samples, fast analysis with low cost, etc.

CE enantioseparation can be achieved in indirect or direct mode. Indirect separation of enantiomers involves the formation of diastereomeric derivatives using chiral derivatization reagents before the $\mathrm{CE}$ separation. This technique needs to have a suitable derivatization reagent with a $100 \%$ enantiomeric purity (if this is not the case, the quantitative analysis will be unsatisfactory) and corresponding functional groups have to be present in the analyte. In addition, care must be taken that no racemization takes place during the derivatization process. Consequently, direct separation mode which allows the separation of unmodified enantiomers in presence of a chiral selector either dissolved in the background electrolyte (BGE) or bound to the capillary wall or immobilized into a gel is often preferred. The most popular and simple mode is the addition of a chiral selector to the free buffer solution. The chiral selector is forming labile diastereoisomers with analytes during the electrophoretic run. Major chiral selectors currently used in CE include neutral and charged cyclodextrins and their derivatives [1-3], crown ethers [4], chiral imprinted polymers [5], enantioselective metal complexes [6], chiral surfactants [7], proteins [8], polysaccharides [9], chiral ion-pairing reagents [10] and macrocyclic antibiotics [11-34].

Macrocyclic antibiotics are the newest class of chiral selectors recently introduced by Armstrong and co-workers [11-24,27,30]. More enantiomers were resolved with these selectors in CE than with any other class of chiral additives in the past few years. Few new chiral selectors have had an 
immediate and dramatic impact as has these macrocyclic antibiotics. The aim of this paper is to present the state of the art about the use of this new class of chiral selectors in CE mainly emphasizing glycopeptide antibiotics which have given the most successful enantioseparations.

\section{Structure and physico-chemical properties}

There are two basic types of macrocyclic antibiotics used in CE. The first type consists of ansamycins which are particulary adept at resolving cationic racemates and the second type is made up of glycopeptides which best resolve anionic analytes. Their chemical structures are shown in figure 1.

The ansamycins, rifamycin $\mathrm{B}$ and rifamycin SV, have a characteristic ansa structure including a ring structure (chromophore) spanned by a highly substituted aliphatic bridge. Rifamycin B differs from rifamycin SV by an R group attached to the naphthohydroquinone ring at position 9 . The $\mathrm{R}$ group is a hydroxyl $(-\mathrm{OH})$ for rifamycin $\mathrm{SV}$ whereas it is an oxyacetic acid $\left(-\mathrm{OCH}_{2} \mathrm{COOH}\right)$ for rifamycin $\mathrm{B}$. Both compounds have nine stereogenic centers. On the aliphatic chain, there are one amide bond, two hydroxyl groups, an ethyl ester on $\mathrm{C}_{21}$ and a methyl ether on $\mathrm{C}_{23}$.

The glycopeptide macrocyclic antibiotics vancomycin, teicoplanin and ristocetin A contain a peptide core of complex amino acids and linked phenolic moieties, one or more neutral sugar moieties, and one or more aminosaccharides. The aglycon portion of fused macrocyclic rings has a semirigid "basket" shape. They differ structurally in a number of ways. Vancomycin has three macrocyclic rings, while teicoplanin and ristocetin A possess four ones. The aglycons of vancomycin and teicoplanin contain 2 chloro-substituted aromatic rings, but the analogous portion of ristocetin $\mathrm{A}$ has no chloro-substituents. All three of the macrocyclic glycopeptides contain an amine on the aglycon portion. Both vancomycin and ristocetin A have one amino saccharide moiety, but teicoplanin has two amino saccharides and both of which are $\mathrm{N}$-acetylated. Moreover, the aglycon portions of vancomycin and teicoplanin have a carboxylic acid moiety, whereas in ristocetin A this group is esterified. All other ionisable groups on these compounds are the phenolic moieties. Of the three macrocyclic glycopeptides, vancomycin containing 18 stereogenic centers is the smallest; ristocetin A, with 38 stereogenic centers, is the largest; while teicoplanin, possessing 23 stereogenic centers, is medium. These
A

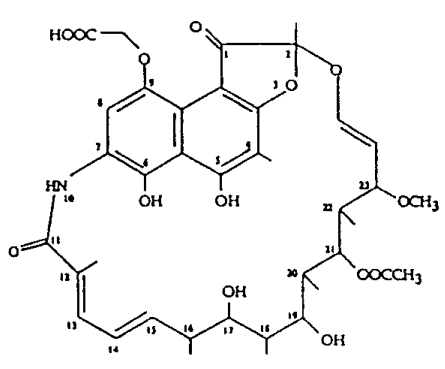

B

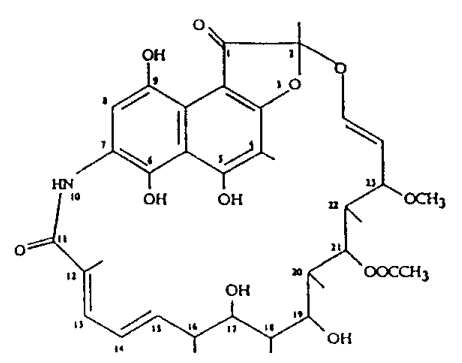

C

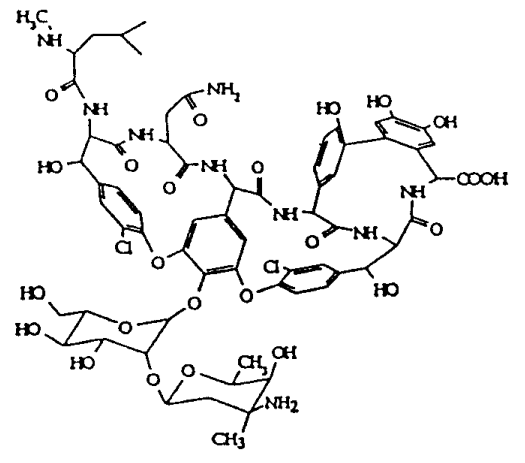

D

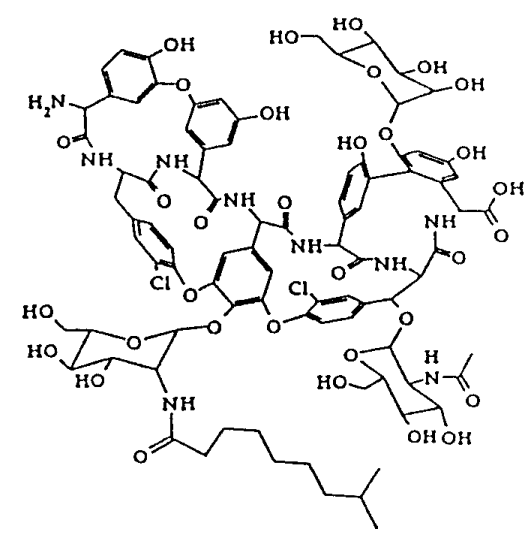

$\mathbf{E}$

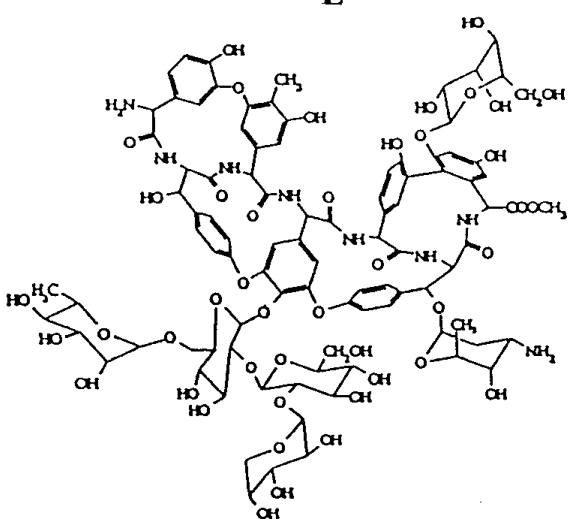

Figure 1. Structures of the macrocyclic antibiotics: A) rifamycin B, B) rifamycin SV, C) vancomycin, D) teicoplanin, E) ristocetin A (reprinted from Ref. [30]. 
structural differences provide a means for altering the selectivity during the chiral separation.

Both rifamycins and glycopeptides absorb strongly in the UV regions. Generally, indirect UV detection is used with rifamycin $\mathrm{B}$ and $\mathrm{SV}$ because of their high concentration used $(2-25 \mathrm{mM})$ in the running buffer.

Rifamycin B is slightly soluble in water. The solubility increases in the short alcohols and acetone. Rifamycin B and SV give stable yellow to orange solutions depending in part on $\mathrm{pH}$ and organic cosolvents [12,26]. Rifamycin B is a diacid with $\mathrm{pK}_{\mathrm{a}} \mathrm{s}$ of approximately 2.8 and 6.7. Consequently it exists as an anion at standard $\mathrm{CE}$ operating $\mathrm{pH}$. Rifamycin $\mathrm{SV}$ is often in neutral form at the same $\mathrm{pH}$.

The macrocyclic glycopeptide antibiotics are soluble in buffers, acidic aqueous solutions and less soluble at neutral $\mathrm{pH}[18,21]$. They are relatively insoluble in most organic solvents. All of glycopeptide selectors tend to degrade with time under conditions commonly used in CE. Degradation usually results in an increase in migration times, baseline noise and a decrease in enantiomeric resolution. The stability of the glycopeptides appears to increase with an increase in the number of attached saccharide moieties. Vancomycin seems to be the least stable macrocyclic antibiotic. Its solutions between pH 5-7 deteriorate within 6-7 days when stocked at $4{ }^{\circ} \mathrm{C}$. Teicoplanin solutions in the same $\mathrm{pH}$ range are somewhat more stable. The most hydrolytically stable macrocyclic antibiotic appears to be ristocetin A in solution, which can be used up to 4 weeks when certain precautions are taken [21]. The glycopeptide solutions should be refrigerated at $4{ }^{\circ} \mathrm{C}$ overnight or when not in use. Under high temperature $\left(\geq 35^{\circ} \mathrm{C}\right)$ as well as $\mathrm{pH}$ outside $4-7$ range, the stability of glycopeptide antibiotics can be greatly reduced. For example, the stabilities of vancomycin, teicoplanin and ristocetin A solutions kept at room temperature $\left(22^{\circ} \mathrm{C}\right)$ were reduced to 2-3 days, 3-4 days and 6-7 days, respectively. Some physico-chemical properties of the three glycopeptide antibiotics are gathered in table I [30].

\section{Enantioselective mechanism}

As previously mentioned, macrocyclic antibiotics contain multiple stereogenic centers. In addition, they have multiple hydrogen bonding groups (hydroxyl and amine), aromatic moieties and hydrophobic clefts or pockets. Furthermore, the groups are in close proximity to one another. Thereby simultaneous interactions needed for chiral recognition are provided.

The primary interaction between the glycopeptide chiral selectors and the analytes is thought to involve chargecharge or ionic interactions $[15,18,21,22]$. Hydrogen bonding, steric repulsion, hydrophobic, dipole-dipole and $\pi-\pi$ interactions are often considered as secondary interactions. In a recent mechanistic study of vancomycin as a chiral selector in CE, Nair et al. [27] concluded that the secondary
Table I. Physico-chemical properties of glycopeptide antibiotics vancomycin, ristocetin $A$ and teicoplanin.(Reprinted from Ref. [30]).

\begin{tabular}{lccc}
\hline Characteristic & Vancomycin & Ristocetin A & Teicoplanin \\
\hline Molecular weight & 1449 & 2066 & 1877 \\
No. of stereogenic centers & 18 & 38 & 23 \\
Produced from & Streptomyces & Norcardia & Actinoplanes \\
fermentation product of & orientalis & lurida & teicomyceticus \\
No. of macrocycles & 3 & 4 & 4 \\
No. of monomer sugar & 2 & 6 & 3 \\
moieties & & & \\
Hydrophobic tail & 0 & 0 & 1 \\
No. of OH groups & 9 & 21 & 15 \\
No. of amine groups & 2 & 2 & 1 \\
No. of carboxylic acids & 1 & 0 & 1 \\
No. of amido groups & 7 & 6 & 7 \\
No. of aromatic groups & 5 & 7 & 7 \\
Methyl esters & 0 & 1 & 0 \\
pI & 7.2 & 7.5 & $4.2,6.5$ \\
Relative stability & $1-2$ weeks & $3-4$ weeks & $2-3$ weeks \\
Current cost & $\$ 65 / g$ & $\$ 1600 / g$ & $\$ 78 / g$ \\
Aggregational behavior & No & No & Yes \\
\hline
\end{tabular}

amine moiety on the aglycon "basket" plays a key role in the enantiorecognition of acidic analytes.

\section{Effect of experimental parameters on chiral resolution}

\section{pH and buffer composition}

The $\mathrm{pH}$ of the background electrolyte (BGE) is a very important parameter to be considered. In fact, the $\mathrm{pH}$ of the running electrolyte governs not only the charge and migration behaviour of the analytes but also that of the chiral selectors. Figure 2 shows the effect of the $\mathrm{pH}$ on electropherotic mobility of vancomycin, teicoplanin and ristocetin $\mathrm{A}$ in a $0.1 \mathrm{M}$ phosphate solution. All of three glycopeptide antibiotics can be both positively or negatively charged according to the $\mathrm{pH}$ of BGE. The curves of vancomycin and ristocetin $\mathrm{A}$ are similar to one another showing a zero mobility (isoelectric point) at $\mathrm{pH} 7.2$ and $7.5(\mathrm{pI})$, respectively. Thus, at $\mathrm{pH}$ range of $4-7$, such as those commonly employed in $\mathrm{CE}$ separations, both vancomycin and ristocetin $\mathrm{A}$ have an overall positive charge. Teicoplanin shows a different behavior exhibiting an anionic character even at acidic $\mathrm{pH}$ ( $\mathrm{pI}=3.8$ ) [21]. As stated previously, rifamycin B is a diacid with $\mathrm{pK}_{\mathrm{a}}$ of 2.8 and 6.7. Hence, both teicoplanin and rifamycin $\mathrm{B}$ migrate, at the $\mathrm{pH}$ range commonly used in $\mathrm{CE}$, against the EOF and in opposite direction to vancomycin and ristocetin. While rifamycin $\mathrm{SV}$ is a neutral chiral 


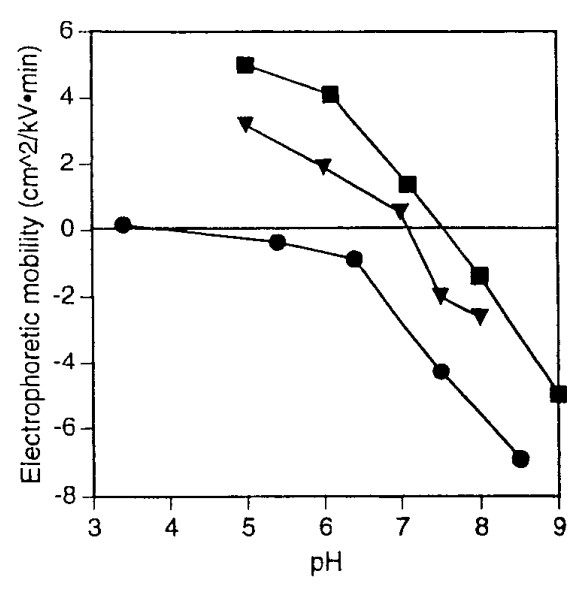

Figure 2. Plot showing the effect of solution $\mathrm{pH}$ on the electrophoretic mobility of ristocetin $A(\square)$, vancomycin $(\mathbf{\Delta})$, and teicoplanin (O) macrocyclic antibiotics using $0.1 \mathrm{M}$ phosphate buffer. The capillary for ristocetin $A$ and vancomycin studies was $32.5 \mathrm{~cm} \times 50 \mu \mathrm{m}$ i.d. $(25 \mathrm{~cm}$ to the detector window). The voltage was $+5 \mathrm{kV}$. The electrophoretic mobility of teicoplanin was obtained using a $44 \mathrm{~cm} \times 50 \mu \mathrm{m}$ i.d. capillary $(36.5 \mathrm{~cm}$ to the detector) and a run voltage of $+10 \mathrm{kV}$. Either acetone or methanol was used as the electroosmotic flow marker. (Reprinted from Ref. [21]).

selector in the same conditions. It is well known that maximum enantioselectivities are most often achieved when the electromigrations of the chiral selector and chiral solutes are opposite to one another [15,29]. Therefore, for rifamycin B, the enantioresolution of an amine-containing analyte increased with increasing $\mathrm{pH}$ and with a maximum at $\mathrm{pH} 7$ where the selector is negatively charged and the analyte is positively charged. Enhanced enantioseparations by vancomycin and ristocetin were found at $\mathrm{pH}$ values slightly below their pI values, 7.2 and 7.5, respectively. However, optimum enantioseparations using teicoplanin as a chiral selector were achieved at $\mathrm{pH}$ values above its $\mathrm{pI}(=3.8)$ [21]. It is important to underline that the change of the $\mathrm{pH}$ has to be limited to the range of 4-7 in order to avoid a rapid degradation of the antibiotics.

The chemical composition and the concentration of the background electrolyte can affect the mobility of the buffer and enantioseparation efficiency when using glycopeptides as chiral selectors. Increasing the concentration of the background electrolyte, i.e., increasing ionic strength of the buffer, results in decrease of separation time. This is due to the decrease of interactions between the glycopeptides and the capillary wall. However, increasing the concentration of the background electrolyte causes an increase in Joule heating. It is well known that Joule heating decreases the efficiency of CE separation. Furthermore, the increase of Joule heating can entail the degradation of glycopeptide antibiotics, since their stability decreases substantially at temperatures above $35{ }^{\circ} \mathrm{C}$. In order to limit Joule heating increase, one can use somewhat higher than normal background elec- trolyte concentrations and lower than normal run voltage to which Joule heating is proportionally related [18,22].

\section{Concentration of macrocyclic antibiotics}

In general, the increase of chiral selector concentration entails an increase of both enantioselectivity and migration time $[12,18,21,26,28]$. For the chiral selector family of the ansamycins such as rifamycin $\mathrm{B}$, the effect of its concentration on migration time is easy to understand. In fact, since the charge on rifamycin $\mathrm{B}$ is negative and the analyte is positively charged at the $\mathrm{pH}$ used in $\mathrm{CE}(\mathrm{pH} 4-7)$, the chiral selector migrates towards the anode (inlet) and the analyte migrates towards the cathode (outlet). The increase of rifamycin B concentration causes an increase of the degree of complexation between analyte and chiral selector. Thus the migration time of the analyte increases. When it comes to glycopeptide antibiotics such as vancomycin and ristocetin $\mathrm{A}$, it might be expected that the migration time of the analyte should decrease as chiral selectors concentration is increased when working with uncoated capillary (so in presence of EOF). Since in contrast with rifamycin B, these two glycopeptide antibiotics are positively charged at the $\mathrm{pH}$ commonly used in CE ( $\mathrm{pH} 4-7)$, while the analytes are negatively charged. However, the migration time decrease has not been observed with any of the glycopeptides studied $[15,18,21]$. The explanation for this observation is that glycopeptide antibiotics adsorb onto the wall of the capillary due to their free amine moieties and the EOF is reduced. Furthermore, once adsorbed to the capillary wall they act as a pseudostationnary phase which interacts with analytes and their migration is retarded. Generally, for a given analyte, migration time with vancomycin is twice that with ristocetin $\mathrm{A}$, while teicoplanin results in intermediate migration time.

\section{Organic modifiers}

The effect of organic modifiers varies according to the type of antibiotics used and analytes to separate. In general, for chiral selectors such as rifamycin B, rifamycin SV and teicoplanin which possess surfactant-like properties, miscible organic modifiers are needed to obtain or enhance the enantioseparation $[12,26,30]$. The reason is thought, in part, that added organic modifiers can inhibit the self-aggregation of these macrocyclic antibiotics which tends to decrease the chiral recognition. The greatest enhancement in enantioresolution occurred with 2-propanol and increasing the 2-propanol concentration increased resolution, decreased electrophoretic mobilities, and thereby increased migration times [12]. The effect of added organic modifiers are usually weak with vancomycin and ristocetin A. Many times the enantioseparation is worse when organic modifiers are added. The cases where weak improvements are observed are believed to be due to slowing of the EOF and/or altering the wall binding behavior of these two glycopeptides. 


\section{Micellar phase}

A mixed micelle system is obtained by adding surfactants such as SDS (sodium dodecylsulfate) above its critical micelle concentration (CMC) to the run buffers containing antibiotics. To date, only the glycopeptide antibiotics have been used in conjunction with micelles. In the case of vancomycin, it is estimated that approximately $90 \%$ of vancomycin is bound to the SDS micelle. Thus there are three pseudophases: the free vancomycin, the vancomycin-SDS mixed micelle and the bulk aqueous solution. The use of such mixed micelle systems allow the resolution a variety of hydrophobic, neutral analytes, e.g., pesticides and drugs [16]. The major separation mechanism is thought to be the partitioning of hydrophobic, neutral compounds between the three pseudophases. In a recent study, Rundlett and Armstrong [17] found the addition of SDS micelles with vancomycin to the run buffer can reverse the elution order of enantiomers, decreases analysis times and enhances efficiencies by one order of magnitude.

The addition of SDS to a teicoplanin-based separation produces similar effects to vancomycin experiments $[17,22]$. However, the elution order of enantiomers in teicoplanin system is not universal. In the case of ristocetin A, the addition of SDS to the analogous conditions does not produce the extensive increase in efficiency and decrease in migration times. Furthermore, there is a significant concentrationdependent effect on selectivity and the reversal of elution order is uncommon [21].

\section{Complementarity among glycopeptide antibiotics used as chiral selectors}

Although all of three glycopeptide antibiotics vancomycin, teicoplanin and ristocetin A belong to the same family, they have some distinct structural differences as well. Therefore, they exhibit somewhat similar but not identical enantioselectivities. In fact, the glycopeptide antibiotics are complementary to one another. Armstrong et al. proposed that if only a partial enantioresolution can be obtained with one glycopeptide, there is a high probability that a baseline or better separation may be obtained with another glycopeptide [30]. This "principle of complementary separations" is illustrated in figure 3 . While it is not yet possible to predict which glycopeptide antibiotic will provide the best resolution for a given analyte, it appears that a partial resolution with one selector usually can be improved upon by using one of the structurally related macrocyclic glycopeptide chiral selectors.

\section{Applications}

Generally, rifamycin B is chosen to separate cationic compounds [12,26], while the glycopeptide antibiotics vancomycin, teicoplanin and ristocetin $\mathrm{A}$ are used for the
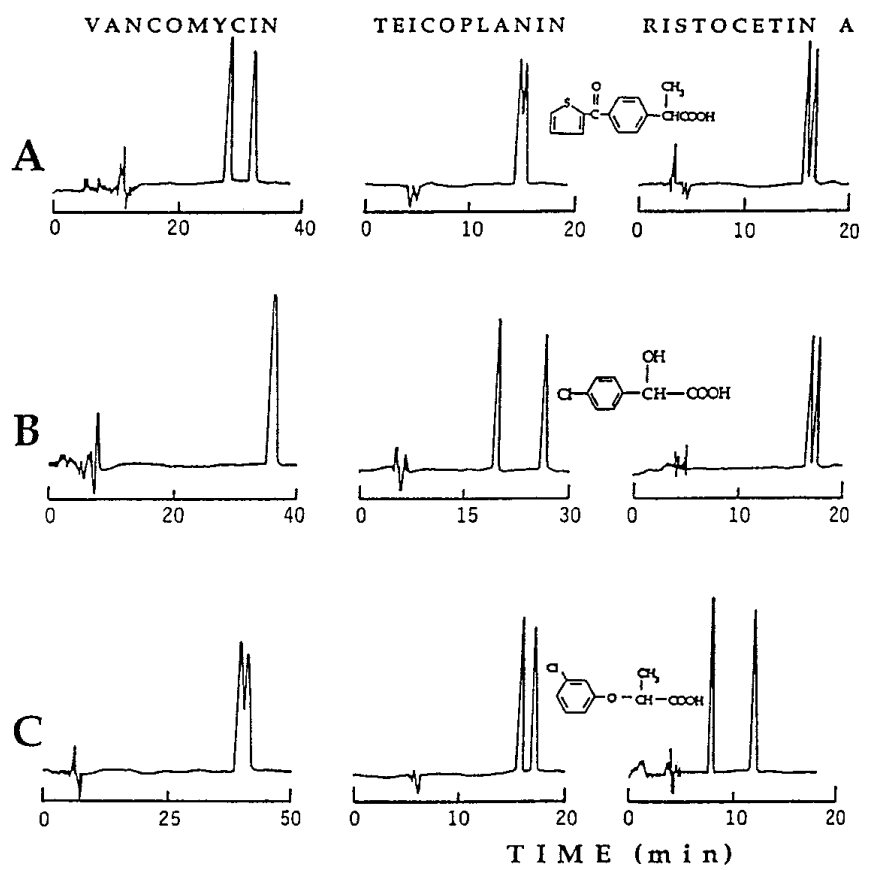

Figure 3. Illustration of the "principle of complementary separations" for the macrocyclic glycopeptide chiral selectors (vancomycin, teicoplanin and ristocetin A). Since they have similar but not identical structures, they tend to have similar but not identical enantioselectivities. Thus if a partial or poor CE separation is obtained with one of the glycopeptides, an improved separation is usually found with one of the related chiral selectors. This is demonstrated by comparing (from left to right the CE separations for three racemates (A) suprofen, (B) p-chloromandelic acid, and (C) 2-(3-chlorophenoxy) propionic acid using the indicated glycopeptides as run buffer additives. Experimental conditions for these separations are given in $[15,18,22,29]$. (Reprinted from Ref. [30]).

separation of compounds containing an anionic moiety such as carboxylate, phosphate, sulphonate groups, etc. Approximately 500 racemic analytes have been electrophoretically resolved with glycopeptide antibiotics, including most $N$-blocked amino acids, numerous nonsteroidal antiinflammatory compounds, antineoplasics, lactic acids, herbicides, rodenticides and a variety of pharmaceutically important compounds $[15,17,18,21,22,28]$. Resolution values between 10 and 20 are not uncommon. For example, a resolution > 22 was obtained for the herbicide 2-(3-chlorophenoxy)propionic acid [18].

The most commonly used glycopeptide in CE is vancomycin because of its availability and relatively low price. However, ristocetin A may be the most widely applicable glycopeptide. In fact, ristocetin A usually produces the shortest migration times, its relative stability in solution is good, it binds the least to the capillary wall and it tends to be the easiest to use [18,21]. Unfortunately it is extremely expensive $(\$ 1600 / \mathrm{g})$. In general, ristocetin A can resolve about as many racemates in $\mathrm{CE}$ as vancomycin and teicoplanin 
combined [15,18,28,31]. A collection of the separations of 120 racemic compounds is given in [18].

\section{New developments}

Recently, two other members of glycopeptide antibiotics, actaplanin A and A82846B, have been evaluated as chiral selectors in CE and have been shown to be successful in achieving enantioseparations $[32,33]$.

Actaplanin A is an analogue of ristocetin A. They differ only in their content and distribution of attached sugar units. The structure of actaplanin A is shown in figure 4.

The chiral selectivity of actaplanin was evaluated as a function of the run buffer $\mathrm{pH}$, chiral selector concentration and organic modifier nature and concentration using six nonsteroidal anti-inflammatory drugs as the test analytes (ketoprofen, flurbiprofen, carprofen, fenoprofen, suprofen and indoprofen) [32]. The authors demonstrated that the organic modifier nature used was very important in altering the enantioselectivity for this macrocyclic antibiotic chiral selector. Among three organic modifiers chosen (methanol, acetonitrile and 2-methoxyethanol), 2-methoxyethanol gives the best overall results. The 2-methoxyethanol behaviour compared to other alcohols may be due to the presence of the methoxy group which increases the polarity of this molecule and in turn may result in the enhancement of charge to charge interactions within the system. All of the enantiomers of the test analytes were baseline separated requiring only $15-30 \%$ of 2 -methoxyethanol, as well as, the use of only a very small amount of the chiral selector actaplanin A $(0.5 \mathrm{mM})$ which allowed a direct detection at $254 \mathrm{~nm}$ (very low background absorbance).

The macrocyclic antibiotic A82846B is an analogue of vancomycin [34]. It differs from vancomycin in two ways: (1) its disaccharide amino sugar is epimeric and (2) it contains an additional epi-vancosamine group. The structures of vancomycin and A82846B are represented in figure 5. A82846B is very soluble in water. It has an isoelectric point at $\mathrm{pH} 9$, reflecting its basic character, and the additional amine group results in a greater positive fractional charge at pHs commonly employed in CE (pH 4-7).

As other macrocyclic antibiotics, A82846B absorbs strongly in UV at low wavelength. Therefore, detection sensibility can be an issue when developing methods for compounds lacking chromophore groups. To overcome this UV interference problem, Reilly and co-workers [33] used a novel countercurrent $\mathrm{CE}$ process, developed by Ward et al. [28], for the evaluation of A82846B as a chiral selector using three dansyl amino acids (dansyl glutamic acid, dansyl serine, dansyl threonine), three anti-inflammatory drugs (flurbiprofen, ketoprofen, suprofen) and the 2-amino-3-(3hydroxy-5-methylisoxazol-4-yl)propionic acid antagonist (LY215490) as test analytes.

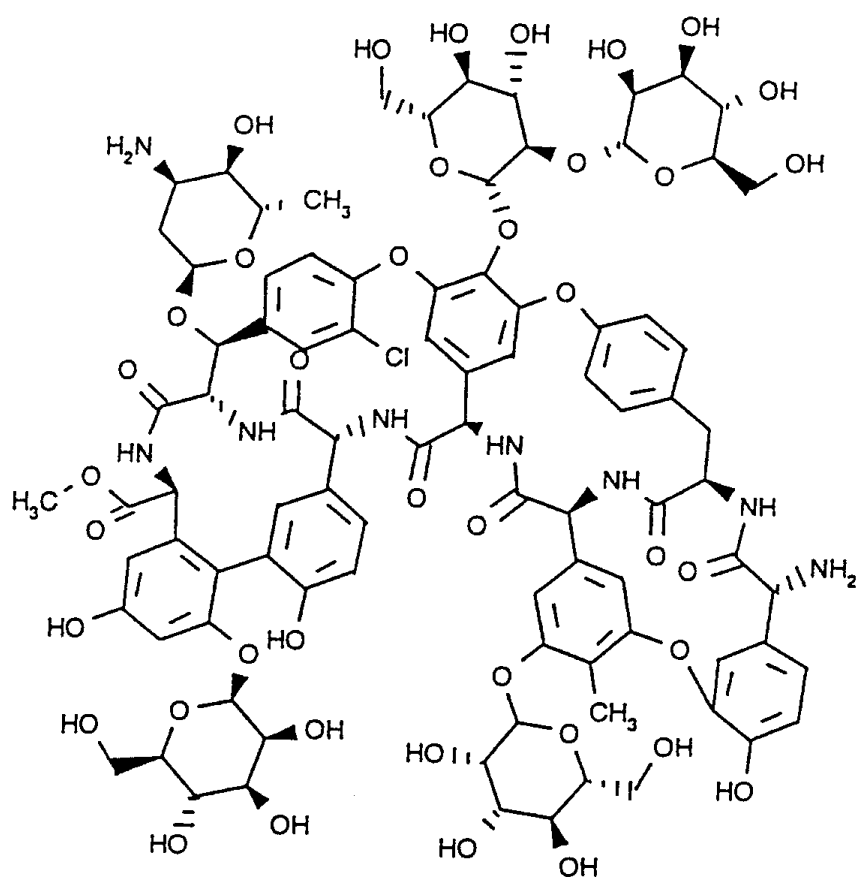

Figure 4. The structure of actaplanin A (Reprinted from Ref. [32]).

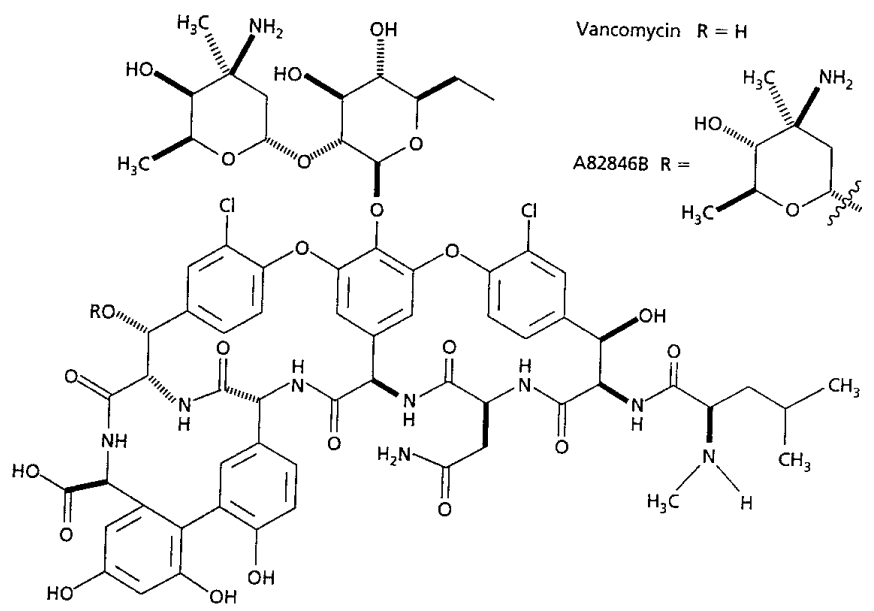

Figure 5. The structure of A82846B and vancomycin. (Reprinted from Ref. [33]).

The principle of the countercurrent CE process is shown in figure 6 . A $27 \mathrm{~cm} \times 50 \mu \mathrm{m}$ desactivated fused-silica capillary (absence of EOF) is filled for 5 min with a run buffer containing A82846B (Fig. 6a) and then a potential of $10 \mathrm{kV}$ is applied across the capillary for $5 \mathrm{~min}$ to ensure the macrocyclic antibiotic would clear the detection cell before injecting samples (Fig. 6b). Then the samples are injected. After 


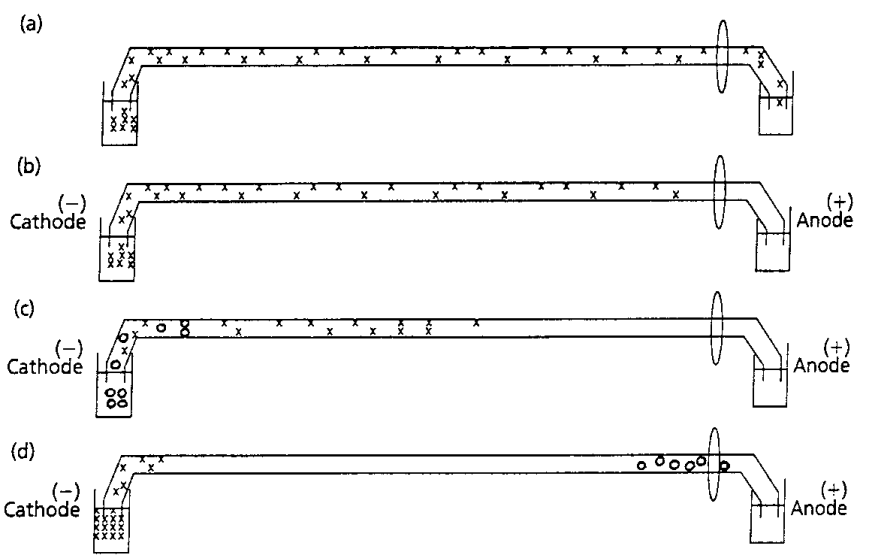

Figure 6. Schematic diagram of the countercurrent CE process used for evaluating A82846B as a chiral selector in which (a) the capillary is filled with run buffer containing A82846B, (b) a $10 \mathrm{kV}$ potential is applied and the A82846B molecules migrate toward the cathode, (c) the sample is injected and the analyte and A82846B molecules migrate in opposite directions, and (d) the separated enantiomers reach the detection cell. (Reprinted from Ref. [33]).

injection, a potential of $10 \mathrm{kV}$ is again applied across the capillary. The positively charged A82846B migrates towards the cathode (the injection end), and the negatively charged analytes migrate towards the anode where the detection is realized (Fig. 6c). Separation is achieved and the analytes reach the detection cell after the chiral selector has cleared the window, thus removing any background absorbance caused by the macrocyclic antibiotic A82846B (Fig. 6d). This approach is a variation of a technique known as partialfilling electrophoresis $[35,36]$. It is important to note that the use of higher concentration of macrocyclic antibiotics in the running buffer is not precluded with this technique since no chiral selector interference exists at the detection window.

By using this technique and after optimizing operating conditions such as buffer $\mathrm{pH}$ and antibiotic concentration, the authors demonstrated that A82846B had provided high resolution of all the enantiomers for the analytes tested in this study. This demonstrates that A82846B is another successful macrocyclic antibiotic that can be used effectively as a chiral selector in $\mathrm{CE}$.

The overall importance of the countercurrent CE process is demonstrated by the baseline separation and detection at $200 \mathrm{~nm}$ of the enantiomers of 2-amino-3-(3-hydroxy-5methylisoxazol-4-yl)propionic acid antagonist (LY215490), while the chiral selector A82846B possesses a strong UV absorbance at this wavelength. In addition, compared with vancomycin, the authors found A82846B to offer higher resolution for flurbiprofen, ketoprofen and suprofen. This again indicates that relatively small structural changes in a macrocyclic antibiotic can provide added selectivity for chiral separations.

\section{Conclusion}

Macrocyclic antibiotics especially the glycopeptides give high enantioselectivity and resolution at very low concentrations towards a wide variety of chiral compounds. That is why they have rapidly become one of the most useful chiral selectors even though they are the newest ones recently developed in CE. Today, CE enantioseparations are dominated by either the cyclodextrin and macrocyclic antibiotic classes of chiral selectors.

However, progress must be realized for overcoming some problems related to the use of macrocyclic antibiotics: adsorption on the capillary wall and degradation with time in the run buffer. Furthermore, without a doubt, much work still needs to be done to fully explore the effects of various parameters on enantioseparation and to understand the underlying enantiorecognition mechanisms when using the macrocyclic antibiotics as chiral selectors. The development of more innovative chiral selectors is to be expected.

\section{References}

1. Fanali, S. J. Chromatogr. A 1997, 792, 227-267.

2. Chankvetadze, B. J. Chromatogr. A 1997, 792, 269-295.

3. Lurie, I. S. J. Chromatogr. A 1997, 792, 297-307.

4. Verleysen, K.; Vandijck, J.; Schelfaut, M.; Sandra, P. J. High Resol. Chromatogr. 1998, 21, 323-331.

5. Lin, J. M.; Nakagama, T.; Wu, X. Z.; Uchiyama, K.; Hobo, T. Fresenius'J. Anal. Chem. 1997, 69, 357-362.

6. Horimai, T.; Ohara, M.; Ichinose, M. J. Chromatogr. A 1997, 760, 235-244.

7. Mechref, Y.; El Rassi, Z. J. Chromatogr. A 1996, 724, 285296.

8. Lloyd, D. K., Aubry, A. F., De Lorenzi, E., J. Chromatogr. A 1997, 792, 349-369.

9. Nishi, H. J. Chromatogr. A 1997, 792, 327-347.

10. Bjornsdottir, I.; Hansen, S.H.; Terabe, S. J. Chromatogr. A 1996, 745, 37-44.

11. Armstrong, D. W.; Tang, Y.; Chen, S.; Zhou, Y.; Bagwill, C.; Chen, J.R. Anal. Chem. 1994, 66, 1473-1484.

12. Armstrong, D. W.; Rundlett, K.L.; Reid, G.L. Anal. Chem. 1994, 66, 1690-1695.

13. Armstrong, D. W. Pittsburgh Conference Abstracts 1994, p 572.

14. Armstrong, D. W.; Zhou, Y. J. Liq. Chromatogr. 1994, 17, 1695-1707.

15. Armstrong, D. W.; Rundlett, K.L.; Chen, J.R. Chirality 1994, 6, 496-509.

16. Armstrong, D. W.; Rundlett, K. L. J. Liq. Chromatogr. 1995, $18,3659-3674$.

17. Rundlett, K. L.; Armstrong, D. W. Anal. Chem. 1995, 67, 2088-2095.

18. Armstrong, D. W.; Gasper, M. P.; Rundlett, K. L. J. Chromatogr. A 1995, 689, 285-304.

19. Armstrong, D. W.; Liu, Y.; Ekborg-Ott, K. H. Chirality 1995, 7, 474-497.

20. Rundlett, K. L.; Armstrong, D. W. J. Chromatogr. A 1996, 721, 173-186. 
21. Gasper, M. P.; Berthod, A.; Nair, U. B.; Armstrong, D. W. Anal. Chem. 1996, 68, 2501-2514.

22. Rundlett, K. L.; Gasper, M. P.; Zhou, Y.; Armstrong, D. W. Chirality 1996, 8, 88-107.

23. Berthod, A.; Liu, Y.; Bagwill, C.; Armstrong, D. W. J. Chromatogr. A 1996, 731, 123-137.

24. Nair, U. B.; Rundlett, K. L.; Armstrong, D. W. J. Liq. Chromatogr. 1997, 20, 203-216.

25. Ward, T. J.; Oswald, T. M. J. Chromatogr. A 1997, 792, 309325 .

26. Ward, T. J.; Dann, C.; Blaylock, A. J. Chromatogr. A 1995, 715, 337-344.

27. Nair, U. B.; Chang, S. S. C.; Armstrong, D. W.; Rawjee, Y. Y.; Eggleston, D. S.; Mcardle, J. V. Chirality 1996, 8, 590-595.
28. Ward, T. J.; Dann, C.; Brown, A. P. Chirality 1996, 8, 77-83.

29. Ekborg-Ott, H.; Gahm, K.; Armstrong, D. W. Chiral Conference of the Americas, Cancun, Mexico 1997; Abstract p 37, 47.

30. Armstrong, D. W.; Usha, B. N. Electrophoresis 1997, 18, 2331-2342.

31. Ward, T. J. $L C \cdot G C$ 1996, $14,428-432$.

32. Trelli-Seifert, L.A., Risley, D. S. J. Liq. Chromatogr. 1998, 21, 299-313.

33. Reilly, J.; Risley, D. S. $L C G C$ 1998, 11, 598-606.

34. Streg, M. A.; Huff, B. E.; Risley, D. S. $L C$ GC 1996, 14, 144148.

35. Tanaka, Y.; Terabe, S. J. Chromatogr. A 1995, 694, 277-284.

36. Nelson, W. M.; Lee, C. S. Anal. Chem. 1996, 68, 3265-3269. 